\title{
Modeling Users in Web Transactional Tasks with Behavioral and Visual Exploration Patterns
}

\author{
Areej Al-Wabil and Mashael Al-Saleh \\ Information Technology Department, College of Computer and Information Sciences \\ King Saud University, Riyadh, Saudi Arabia \\ \{aalwabil, malsaleh2\}@ksu.edu.sa
}

\begin{abstract}
In this paper, we describe a model of how users conduct transactional tasks on the web. In an exploratory eye tracking experiment, visual patterns of user interactions with web-based transactions were examined for verifying the model. Findings suggest viewing patterns and interactions supportive of the proposed user model and provide a good match to participants' interactions in completing transactional tasks on Arabic interfaces.
\end{abstract}

Keywords: Eye tracking, transactional tasks, visual attention, user model.

\section{Introduction}

Modeling user behavior facilitates the optimization of interface designs. Services and transactions are increasingly being offered online for users, but there exists limited research on modeling users in transactional tasks when compared to navigational and informational tasks. User modeling is needed to investigate effective methods for optimizing transactional interfaces and facilitating the development of automated usability evaluations. Recently, models have been proposed to investigate navigational and informational tasks in which users search for specific information elements in interactive systems. Navigational models include the framework for Human-Web Interaction [1], theories that have been developed for the purpose of cognitive engineering include Norman's Theory of Action [2], and frameworks which have informed usability practice include the User Action Framework [3].

Eyetracking has been shown to be a viable approach for examining the cognitive and perceptual capabilities of users in their interaction with interfaces [e.g. 4]. The focus of this study involves developing a user model that focuses on transactional tasks on the web. The model is motivated by the Theory of Action [2], which quantifies the perceived relevance of web form elements to the user's goal by cognitive processing and behavioral mechanisms. It utilizes behavioral and eye gaze metrics, derived from visual exploration patterns. In exploratory eye tracking experiments, visual patterns of user interactions with web-based transactions were examined for verifying the model's representation of user behavior and cognition. 


\section{A Model of Interaction in Transactional Tasks}

In this study we propose a behavioral model of human interaction in transactional tasks, particularly web forms. The model can inform the design of a framework for examining usability issues in web forms. The model assumes that users complete transactional tasks in web form sequentially based on behavioral mechanisms that involve perceptual, cognitive and motor responses. It is depicted in Figure 1.

The first stage involves determining the goal for completing the transaction and the intention by ensuring that the form matches the goal of the transaction. After that, users proceed to fill out the form fields by first determining each field's strategy. The Determine Strategy step involves scanning the interface for cues on the form controls to understand how to proceed. The Modifying Form Controls stage is an iterative multi-step process which involves selecting the controls (e.g. field, radio button, checkbox, etc.) and modifying the controls by applying the appropriate action for entering data or selecting elements. Following that, the perceive system state involves scanning the interface to identify feedback and examining the system's response to understand what needs to be done. The evaluate field sub-stage involves understanding the system state to determine whether to proceed or act upon the feedback received from the system. Finally after completing all fields, the Conclude Transaction stage involves scanning form elements before concluding the transaction.

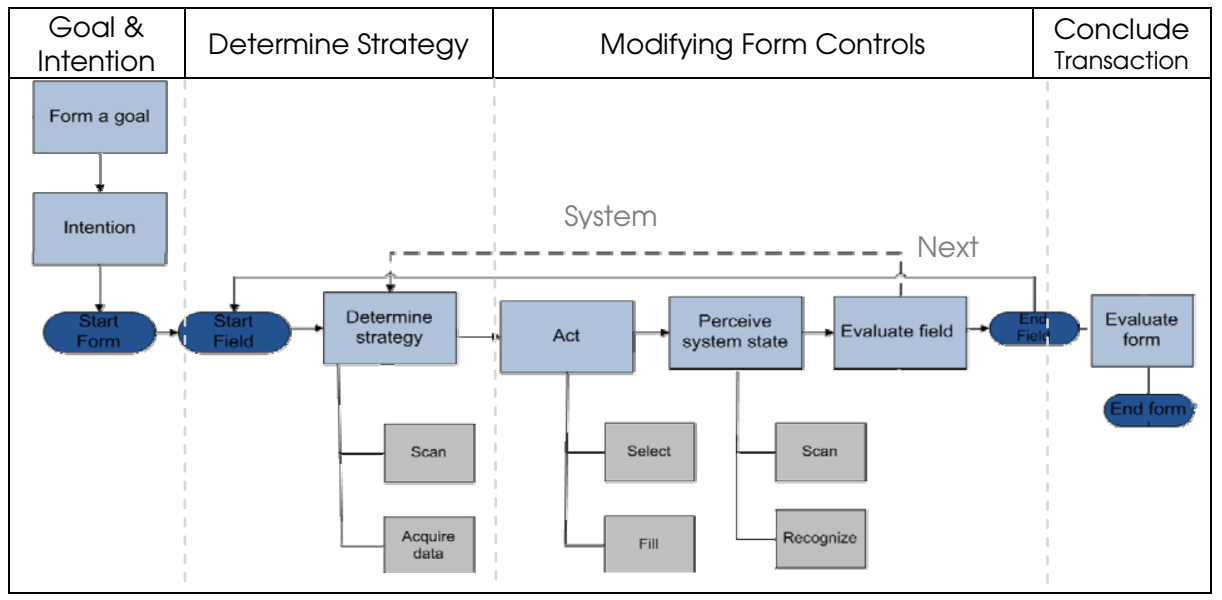

Fig. 1. Proposed model of interaction in transactional tasks

\section{Method}

The exploratory study was conducted in a lab environment controlled for consistent lighting, temperature and sound. The model was tested against a detailed set of eye tracking data collected from ten participants as they engaged in three transactional tasks using Arabic interfaces of web-based forms. 


\subsection{Participants and Apparatus}

Ten participants took part in this exploratory experiment. Participants ranged in age between 17-34 years $($ Mean $(M)=23.4$ years, and Standard Deviation $(\mathrm{SD})=4.5$ years). Their computer experience ranged between 8-14 years of usage (M=10.7 years, $\mathrm{SD}=2.37$ years) and their Internet experience similarly ranged between 7-14 years $(M=9.7, S D=2.45)$. A standalone Tobii X120 eye tracker was used for gaze capture; it samples the position of users' eyes at the rate of $120 \mathrm{~Hz}$ with accuracy of approximately $0.5^{\circ}$. Gaze data were logged by Tobii Studio 2.1. Internet Explorer 7 was used to display web stimuli with a resolution of $1024 \times 768$.

\subsection{Experimental Design: Stimuli and Procedure}

Each participant was asked individually to complete three transactional tasks on Arabic web interfaces so as to fill out forms related to different topics. The web pages participants viewed were selected to be representative of sectors such as public information, business and academic. Tasks involved booking a flight online with prespecified departure and return dates, registering for an online academic portal, and searching for the contact form on a public service web site. Order of transactional tasks was counterbalanced across participants. Two examples are shown in Table 1.

The experiment consisted of three parts. The first part included demographic questions and familiarity with computers and the web. The second part was the main eye tracking study. The third part was the retrospective think-aloud (RTA) protocol on a sub-set of tasks in which participants describe their thoughts as they completed transactional tasks to elicit a deeper understanding of their observed behavior.

Table 1. Sample of stimuli

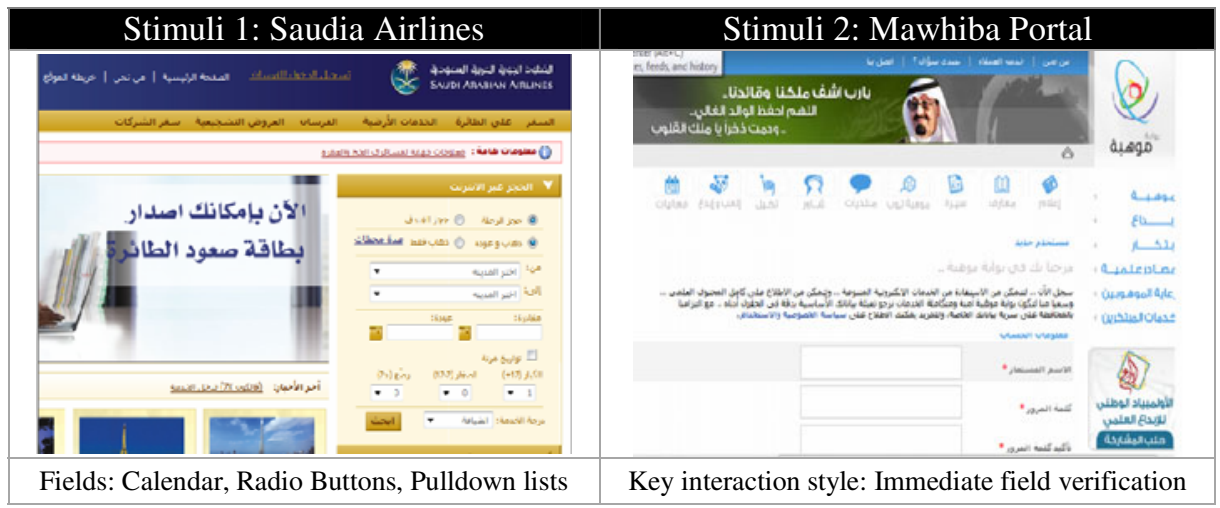

\section{Results and Discussion}

In this study, we examined visual and behavioral strategies in transactional tasks, and present our findings in four parts according to the key stages of the user model. 


\subsection{User Goal and Intentions}

To investigate this part of the model, we examined a navigation task in which users searched an organization's landing page for the contact form. It was evident that links with descriptive titles received more and longer fixation durations than other competing links. Verification of intent and goal matching was exhibited by users in visual explorations of form titles and headers/introductions and supported by RTA verbalizations. An example of a user experiencing difficulties is depicted in Figure 2.

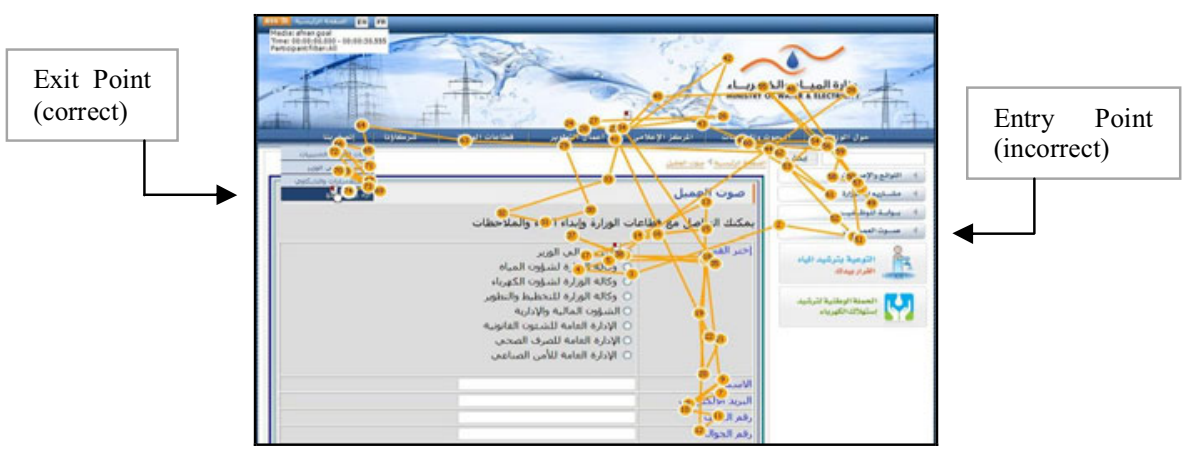

Fig. 2. Scattered scanpaths exhibited for verifying whether a form matches a user's goal

\subsection{Part 2: Determining the Strategy}

Examining patterns of how users determined the strategy for selecting dates on a reservation form, participants varied in familiarity with this form control. Four participants exhibited systematic patterns of examining labels followed by field controls. The RTA confirmed familiarity of participants with this type of field controls. This is depicted in Figure 3.

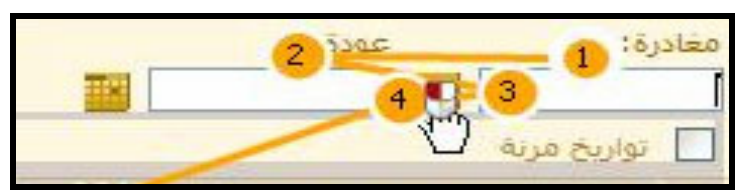

Fig. 3. Visual pattern reflecting the 'Determine Strategy' stage: Users examine the field labels for departure and return flights, then data entry field, then the calendar icon

The remaining participants had no prior experience with calendar controls for fields and exhibited on average more fixations and longer mean fixation durations than the first group. The visual patterns exhibited by this group were either scattered scanpaths with a relatively large number of fixations or intense fewer fixations suggesting increased cognitive processing in trying to figure out how to enter a date. 


\subsection{Modifying Form Controls}

The Act sub-stage involves direct selection of form controls followed by modifying the controls by key presses, mouse clicks or selection of radio buttons etc. The perceive system state sub-stage was interesting to examine with eye gaze data as patterns revealed insights into how users perceive system feedback. For example, the Mawhiba form included immediate verification of fields and thus the user in Figure 4 noticed the error message indicating the problem with the username. Scanpaths show users visually scanning the system state and returning to the field and recognizing the problematic element and acting upon the feedback. Once corrected, users proceeded to the next field as depicted in Figure 4.
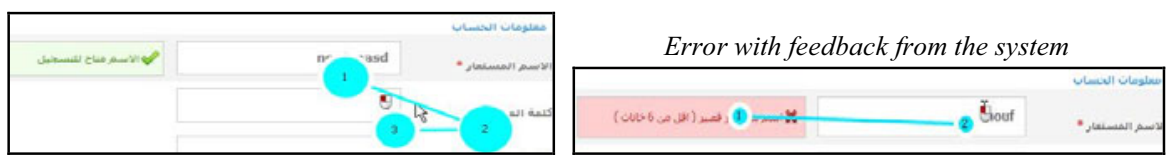

Fig. 4. Modifying Form Controls: Perceiving and Acting upon system state

\subsection{Concluding Transactions}

In evaluating a form before concluding transactions, participants exhibited one of three patterns. The first two were top-down or bottom-up visual scan to verify form elements before submitting. The third was directing attention to the submit control on the form to conclude the transaction without verification.

\section{Conclusion}

Findings show viewing patterns and interactions supportive of the proposed model, and it was concluded that the model provided a good match to users' interactions in completing transactional tasks. Furthermore, insights obtained from the exploratory experiments have led to revising segments of the model's stages as well as establishing a set of predictions for validating the model in future work.

\section{References}

1. Pilgrim, C., Lindgaard, G., Leung, Y.: A Framework for Human-Web Interaction. In: Proceedings of the CHISIG Annual Conference on Human-Computer Interaction, Ergonomics Society of Australia, Wollongong. ACM, New York (2004)

2. Norman, D.: Cognitive Engineering. In: Norman, D.A., Draper, S.W. (eds.) User Centered System Design, pp. 31-62 (1986)

3. Andre, T., Hartson, H., Belz, S., McCreary, F.: The User Action Framework: A Reliable Foundation for Usability Engineering Support Tools. Human-Computer Studies 54, 107-136 (2001)

4. Nielsen, J., Pernince, J.: Eyetracking Web Usability. New Rider, New York (2010) 